Bangladesh J. Plant Taxon. 28(1): 83-95, 2021 (June)

(C) 2021 Bangladesh Association of Plant Taxonomists

https://doi.org/10.3329/bjpt.v28i1.54210

\title{
FILAMENTOUS CYANOBACTERIA FROM WESTERN GHATS OF NORTH KERALA, INDIA
}

\author{
V. Geethu and Mamiyil Shamina ${ }^{1}$ \\ Cyanobacterial Diversity Division, Department of Botany, \\ University of Calicut, Kerala, India
}

Keywords: Cyanobacteria, Filamentous, Peruvannamuzhi, Western Ghats.

\begin{abstract}
Cyanobacteria are Gram negative, photosynthetic and nitrogen fixing microorganisms which contribute much to our present-day life as medicines, foods, biofuels and biofertilizers. Western Ghats are the hotspots of biodiversity with rich combination of microbial flora including cyanobacteria. Though cosmopolitan in distribution, their abundance in tropical forests are not fully exploited. To fill up this knowledge gap, the present research was carried out on the cyanobacterial flora of Peruvannamuzhi forest and Janaki forests of Western Ghats in Kozhikode District, North Kerala State, India. Extensive specimen collections were conducted during South-West monsoon (June to September) and North-East monsoon (October to December) in the year 2019. The highest diversity of cyanobacteria was found on rock surfaces. A total of 18 cyanobacterial taxa were identified. Among them filamentous heterocystous forms showed maximum diversity with 10 species followed by non- heterocystous forms with 8 species. The highest number of cyanobacteria were identified from Peruvannamuzhi forest with 15 taxa followed by Janaki forest with 3 taxa. The non- heterocystous cyanobacterial genus Oscillatoria Voucher ex Gomont showed maximum abundance with 4 species. In this study we reported Planktothrix planktonica (Elenkin) Agagnostidis \& Komárek 1988, Oscillatoria euboeica Anagnostidis 2001 and Nostoc interbryum Sant'Anna et al. 2007 as three new records from India.
\end{abstract}

\section{Introduction}

Cyanobacteria are morphologically distinct group of Gram negative, photosynthetic and nitrogen fixing microorganisms. They are believed to evolve during Proterozoic Era between 2.5 and 3.5 billion years ago commonly known as the Age of Cyanobacteria (Hoek et al., 1993) and these prokaryotes made our planet earth oxygenic (Gupta et al., 2006). They have constituted the most diverse group of organisms in Plant Kingdom. They are ubiquitous and highly versatile to grow in various climatic conditions such as freshwater, marine, hot springs, polar deserts, epilithic, epipelic, epiphytic, endophytic, halophytic and thermophilic conditions (Halder, 2015, 2016). Cyanobacteria are economically important because of their ability to fix atmospheric nitrogen and are used as biofertilizers (Mishra and Pabby 2004). Besides, it excretes various secondary metabolites and bioactive compounds which are useful in various industries such as pharmaceuticals, biofuels, cosmetics, etc. Being rich in phycobiliproteins they are used as natural colorants (Pandey et al., 2013).

Tropical India provides favourable environments for the luxuriant growth of cyanobacteria in her natural ecosystems (Thajuddin et al., 2002; Chellappa et al., 2004). So far, the cyanobacteria of India have been studied by various workers such as Chakraborty et al. (2010), Naskar et al. (2008), Keshri and Chatterjee (2010), Ansari et al. (2012), Bhosale and Dhumal (2012), Tiwari

\footnotetext{
${ }^{1}$ Corresponding author: Email: drshaminam@gmail.com
} 
and Chauhan (2006, 2008), Dhingra and Ahluwalia (2007a,b), Gupta (2012), Haldar and Sinha (2013), Dey (2012), Roy et al. (2014a,b) and Datta and Keshri (2014).

The Western Ghats of India are one of the richest biodiversity hotspot in the world. Western Ghats supports a vast vegetation including 7400 species of flowering plants, 493 species of bryophytes, 320 species of ferns and 750 species of fungi. But for cyanobacterial flora, it is believed that the area is least explored and and as a result many cyanobacterial taxa remains undiscovered. To fulfil this knowledge gaps, the present research was undertaken to explore the diversity of filamentous cyanobacteria in Peruvannamuzhi forest range of Western Ghats region.

\section{Materials and Methods}

The study was conducted in Peruvannamuzhi forest and Janaki forest of Western Ghats located in Kozhikode District, Kerala state, India. This area is a part of Malabar Wild life Sanctuary and this region stretches between $11.5966^{\circ} \mathrm{N}$ and $75.8232^{\circ} \mathrm{E}$ covering an area of $74.215015 \mathrm{~km}^{2}$.

Extensive specimen collection trips were conducted during South-West monsoon (June to September) and North-East monsoon (October to December) in 2019. All specimens were collected using forceps, knife, scalpels, needles, for the habitats like the surfaces of rock, soil, tree bark, etc. For the water bodies or moistened parts plastic bottles were used. All the field observations on habit, habitat and soil $\mathrm{pH}$ were noted in the field book at the time of collection. Sub-samples from the collected specimens were carefully examined under compound microscope (Model-Leica DM 1000 Compound Microscope, Germany). All studied specimens were cultured in BG-11 medium (Rippka et al. 1979). A part of the cultured specimen was preserved in 4\% commercial formaldehyde and deposited in the culture collection at Cyanobacterial Diversity Division, University of Calicut, India.

Classification of cyanobacteria by Komarek et al., (2014) was followed in the present study. Based on cellular morphology, the cyanobacterial individuals were identified up to the species or genus level consulting monographs and floras of Desikachary (1959), Anand (1989), John and Francis (2013) and Komárek and Anagnostidis (2005).

\section{Results and Discussion}

In the present investigation, a total of 18 taxa of filamentous cyanobacteria belonging to the orders Oscillatoriales and Nostocales were recorded. The detailed taxonomic description of those have been given below.

\section{Division: Cyanophyta; Class: Cyanophyceae; Order: Oscillatoriales Family: Microcoleaceae; Genus: Microcoleus Desmazières ex Gomont}

1. Planktothrix planctonica (Elenkin) Anagnostidis et Komárek

(Fig. 1A) (Anagnostidis \& Komarek in Archiv für Hydrobiologie, 80, 416, 1988; Dey et al. in Flora of Australia Supplementary Series 4: i-vii, 1-276, 1995)

Filaments blue green, slightly waved; cells $7.4-8.2 \mu \mathrm{m}$ wide, $2.2-3.8 \mu \mathrm{m}$ long, shorter than broad, slightly or not constricted at cross walls; apical cells widely rounded, 3.0 - 5.2 $\mu \mathrm{m}$ long, 7 $\mu \mathrm{m}$ broad; granules present; sheath absent.

Ecology: Attached scum on stone in stream.

Specimen examined: Kozhikode District, Peruvannamuzhi forest, Kerala, India: 13 Sep 2018, V. Geethu, Cyanobacterial Diversity Division, University of Calicut, CU No: 158535 
Distribution: Kerala, Peruvannamuzhi forest, India; The Netherlands, Romania, Czech Republic; Russia, Tajikistan; South Australia; Pacific Islands; Hawaiian Islands.

Comment: The species has been reported as a first record in India

Family: Oscillatoriaceae; Genus: Oscillatoria Vaucher ex Gomont

2. Oscillatoria princeps Vaucher ex Gomont

(Fig. 1B)

(Vaucher, Historie des conferves d' eau douce, 190, pl. 15, fig. 2, 1803; Gomont, Monogr. Oscillatorees, 206, pl. fig. 9, 1892; Forti in De Toni, Sylloge Algarum, 5 : 150, 1907; Fremy, Myxo. d’ Afr. Equat. Franc., 208, fig. 175, 1929; Geitler, Kryptogamenflora, 947, figs. 598a, 601cg 1932)

Trichome blue green to dark green, straight, not constricted at cross walls, $12.2-14.5 \mu \mathrm{m}$ broad, 2.9-3.5 $\mu \mathrm{m}$ long; end cell flatly rounded or hemispherical, pale yellow, $3.3 \mu \mathrm{m}$ long; granules present; necridia present.

Ecology: Attached to stone.

Specimen examined: Kozhikode District, Peruvannamuzhi forest, Kerala, India: 21 Nov 2018, V. Geethu, Cyanobacterial Diversity Division, University of Calicut, CU No: 158535 .

Distribution: Kerala, Peruvannamuzhi forest, India;The Baltic Sea, Black Sea, Britain, Czech Republic, France, Georgia, Germany, Greece, Ireland, Lithuania, Netherlands, Romania, Scandinavia, Slovakia, Spain; Atlantic Islands: Canary Islands;: Arkansas, Florida, Great Lakes, Mexico, Northwest Territories, Québec; Caribbean Islands: Cuba;Argentina, Brazil; Africa: Ghana, Mozambique, Sierra Leone, Sudan,Mauritius, Bangladesh, India, Iraq, Israel, Kuwait, Pakistan, Punjab, Saudi Arabia, Sri Lanka,China, Japan, Korea, Nepal, Russia, South China Sea, Taiwan, TajikistanThailand, Vietnam, Australia, New Zealand

3. Oscillatoria subbrevis Schmidle

(Fig. 1C) (Engler's Bot. Jahrb., 30, pl. IV [4], fig. 7, 243, 1901; Forti in DeToni, Sylloge Algarum, 5, fig. 174, 208, 1907; Fremy in Myxo. d' Afr. Equat. Franc., fig. 174, 208, 1929; Geitler in Kryptogamenflora, fig. 601b, 949, 1932; Desikachary in Cayanophyta, pl. 37, fig. 2, pl. 40, fig 1, 207, 1959)

Filaments greenish brown, without sheath $10.3-11.9 \mu \mathrm{m}$ broad, with sheath $11.9 \mu \mathrm{m}$ broad; trichome $1.3-1.9 \mu \mathrm{m}$ long, $7.9 \mu \mathrm{m}$ broad, greenish brown; end cell rounded, pale yellow, 2.7 - 4 $\mu \mathrm{m}$ long, $7.9 \mu \mathrm{m}$ broad; sheath colourless, $0.89 \mu \mathrm{m}$ thick.

Ecology: Attached scum on stone.

Specimen examined: Kozhikode District, Janaki forest, Kerala, India: 21 Jan 2019, V. Geethu, Cyanobacterial Diversity Division, University of Calicut, CU No: 167110.

Distribution: Kerala, Janaki forest,India,Britain, Greece, The Netherlands, Romania, Spain,Argentina, Brazil; Africa: Sierra Leone, Sudan,Bangladesh, India, Iran, China, Japan, Korea, Nepal, Singapore, Australia, The New Zealand.

4. Oscillatoria euboeica Anagnostidis

(Fig. 1D) (Anagnostidis in Preslia, Praha 73: 359 - 375, 2001; Temraleeva in Microbiology, 87: 2, tab, 251, 2018)

Filaments light green, $8.2-9.2 \mu \mathrm{m}$ broad; cells short, $3.5-4.1 \mu \mathrm{m}$ long, apex curved, constrictions absent; apical cell rounded, pale yellow $3.2 \mu \mathrm{m}$ long, $6.4 \mu \mathrm{m}$ broad; sheath absent ; granules present. 


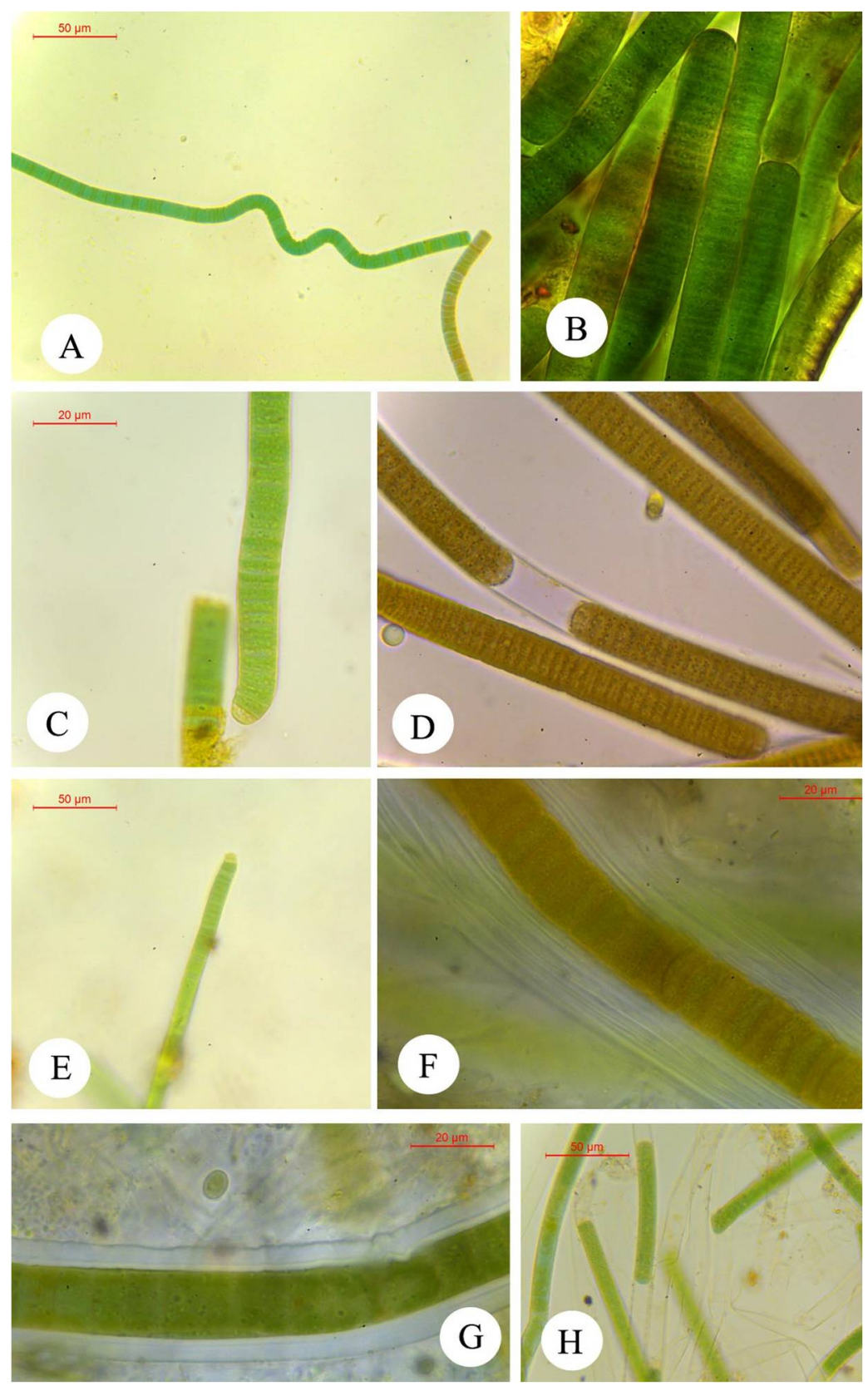

Fig. 1. A. Planktothrix planctonica, B. Oscillatoria princeps, C. O. subbrevis, D. O. euboeica, E. O. limosa F. Lyngbya majuscula G. L. subconfervoides, H. Phormidium retzi var. major.

Ecology: Attached to soil.

Specimen examined: Kozhikode District, Peruvannamuzhi forest, Kerala, India: 13 Sep 2018, V. Geethu, Cyanobacterial Diversity Division, University of Calicut, CU No: 158537. 
Distribution: Kerala, Peruvannamuzhi forest, India.

Comment: The species has been reported as a first record in India.

5. Oscillatoria limosa Agardh. ex Gomont

(Agardh, Dispositio Algarum Sueciae, 35, 1812; Gomont, Mongr. Oscillatorees, 210, pl. 6, fig. 13, 1892; Forti in De Toni, Sylloge Algarum, $5: 154,1907$ )

Filaments straight, light green; trichome not constricted at cross wall, $9.6 \mu \mathrm{m}$ broad, $2-2.5$ $\mu \mathrm{m}$ long; granules present; end cell flatly rounded, pale yellow in colour.

Ecology: Attached to stone.

Specimen examined: Kozhikode District, Peruvannamuzhi forest, Kerala, India: 21 Nov 2018, V. Geethu, Cyanobacterial Diversity Division, University of Calicut, CU No: 158537.

Distribution: Kerala, Peruvannamuzhi forest, India, Svalbard, Baltic Sea, Britain, Channel Islands, Czech Republic, France, Georgia, Germany, Greece, Italy, Lithuania, Norway, Poland, Romania, Russia, Scandinavia, Slovakia, Spain, Sweden, Ukraine, Arkansas Great Lakes, Mexico, Northwest Territories, Québec, Tennessee ,Caribbean Islands, Cuba, Argentina, Brazil, Africa, Egypt, South Africa, SudanBangladesh, India, Iraq, Israel, Saudi Arabia,Caspian Sea, China, Japan, Korea, Nepal, Tajikistan,Vietnam; Australia The New Zealand, Australia, Pacific Islands, , Singapore.

6. Lyngbya majuscula Harvey ex Gomont

(Fig. 1F) (Harvey in Hooker, English Flora, 5, Part 1: 370, 1833; Gomont, Monogr. Oscillatoriees, 151, pl. 3, fig. 3, 4, 1892; Forti in De Toni, Sylloge Algarum, 5: 268, 1907)

Filaments yellowish green, $46 \mu \mathrm{m}$ broad; cells $16 \mu \mathrm{m}$ broad, $4-6 \mu \mathrm{m}$ long; sheath thick, colourless, lamellated, $11.5-12.9 \mu \mathrm{m}$ in thick; granules present.

Ecology: Attached to stone on the stream

Specimen examined: Kozhikode District, Peruvannamuzhi forest, Kerala, India: 21 Nov 2018, V. Geethu, Cyanobacterial Diversity Division, University of Calicut CU No 167101.

Distribution: Peruvannamuzhi forest, Kerala, India; Baltic Sea, Britain, Channel Islands, France, France (Breizh), Greece Ireland, Romania, Russia, Scandinavia, Spain, Canary Islands, Florida, Isla Guadalupe, Mexico, Brazil, Colombia, Venezuela,Djibouti, Egypt, Eritrea, Ethiopia, Kenya, Madagascar, Mauritius, Mediterranean Sea, Mozambique, South Africa, Sudan, Tanzania, Indian Ocean Islands, Aldabra Islands, Chagos Archipelago, Comoros and Mayotte, Laccadive Islands, Maldives, Réunion, Rodrigues; South-west Asia: Bangladesh, India, Iran, Iraq, Kuwait, Pakistan, Saudi Arabia, Sri Lanka, Turkey, Yemen,China, Japan, Korea, Nepal, South China Sea, Taiwan,Indonesia, Malaysia, Philippines, Singapore, Vietnam, Australia, The New ZealandCentral Polynesia, Federated States of Micronesia, French Polynesia, Guam, Hawaiian Islands (HI), Line Islands, Mariana Islands, Marshall Islands, Northwestern Hawaiian Islands, Republic of Palau. fig. 6, 1918; Geitler, Kryptogamenflora, 1067, fig. 681, 1932; Desikachary in Cyanophyta, 321, 1959)

Filaments long, straight, $26.6-35.0 \mu \mathrm{m}$ broad, bluish green, not constricted at cross wall, cross walls not granulated, $16.5 \mu \mathrm{m}$ broad, $6.1-7.3 \mu \mathrm{m}$ long; end cell rounded; sheath thick, colourless, unlamellated, $5.6 \mu \mathrm{m}$ thick; calyptra absent. 
Ecology: Attached to stones on stream

Specimen examined: Kozhikode District, Peruvannamuzhi forest, Kerala, India: 21 Nov 2018, V. Geethu, Cyanobacterial Diversity Division, University of Calicut CU No: 167101.

Distribution: Kerala, Peruvannamuzhi forest, India; China, Japan.

8. Phormidium retzi var. major Kützing ex Gomont

(Fig. 1H)

(Myxophyceae of Travancore State, Proc. Indian Acad. Sci. B, 11 : 122, 1940; Desikachary in Cyanophyta, 268, 1959; Yadhav et al. in Asian J. Environ. Sci., 7: 2, tab. 1, 256, 2012)

Filaments dull blue green, more or less straight, unconstructed at the cross walls, $9.9-10.3$ $\mu \mathrm{m}$ broad; trichome shorter than broad, $9.7-10 \mu \mathrm{m}$ broad, $6.4-7.2 \mu \mathrm{m}$ long; septa not granulated; end cell rounded; sheath extention present, sheath, colourless, unlamellated

Ecology: Seen on soil

Specimen examined: Kozhikode District, Peruvannamuzhi forest, Kerala, India: 21 Nov 2018, V. Geethu, Cyanobacterial Diversity Division, University of CalicutCU No: 158537.

Distribution: Kerala, Peruvannamuzhi forest, India.

Division : Cyanophyta; Class: Cyanophyceae;Order: Nostocales

Family: Scytonemataceae; Genus :Scytonema C.Agardh ex É.Bornet et C.Flahault

9. Scytonema pascheri Bharadwaja

(Fig. 2A)

(Desikachary in Cyanophyta. pp. i-x, 1-686, pls 1-139. New Delhi: Indian Council of Agricultural Research, 1959)

Filaments brown, 19-22.8 $\mu \mathrm{m}$ broad; cells quadrate, $17-21.5 \mu \mathrm{m}$ long, $12-14 \mu \mathrm{m}$ broad; heterocyst intercalary, rounded, $11.8 \mu \mathrm{m}$ long, $12.7 \mu \mathrm{m}$ broad; sheath yellowish brown, lamellated, 2.25-4.3 $\mu \mathrm{m}$ in thickness; granules present.

Ecology: Attached scum on stone

Specimen examined: Kozhikode District, Chembanoda, Kerala, India: 17 Mar 2019, V. Geethu, Cyanobacterial Diversity Division, University of Calicut CU No: 167126.

Distribution: Kerala, Peruvannamuzhi forest, India; China, Bangladesh

Family: Rivulariaceae; Genus: Rivularia C.Agardh ex Bornet et Flahault

10. Microchaete investiens Fremy

(Fig. 2B)

(Fremy in Archives de Botanique, Memoires, 3: 2, fig. 249, 283, 1930; Hirose et al. in Illustrations of Japanese fresh water algae, 1-933, 1977)

Filaments long, brown, 6.1-8.1 $\mu \mathrm{m}$ broad; cells elongated, cylindrical or barrel shaped, 5.7$8.1 \mu \mathrm{m}$ broad, $6.5-11 \mu \mathrm{m}$ long; heterocyst basal or intercalary, 4-6.3 $\mu \mathrm{m}$ long, 5.6-11 $\mu \mathrm{m}$ broad; granules present; sheath colourless to yellowish, $0.94 \mu \mathrm{m}$ in thickness.

Ecology: Attached scum on stone

Specimen examined: Kozhikode District, Peruvannamuzhi forest, Kerala, India: 13 Sep 2018, V. Geethu, Cyanobacterial Diversity Division, University of Calicut, CU No: 158537.

Distribution: Kerala, Peruvannamuzhi forest, India; Japan 

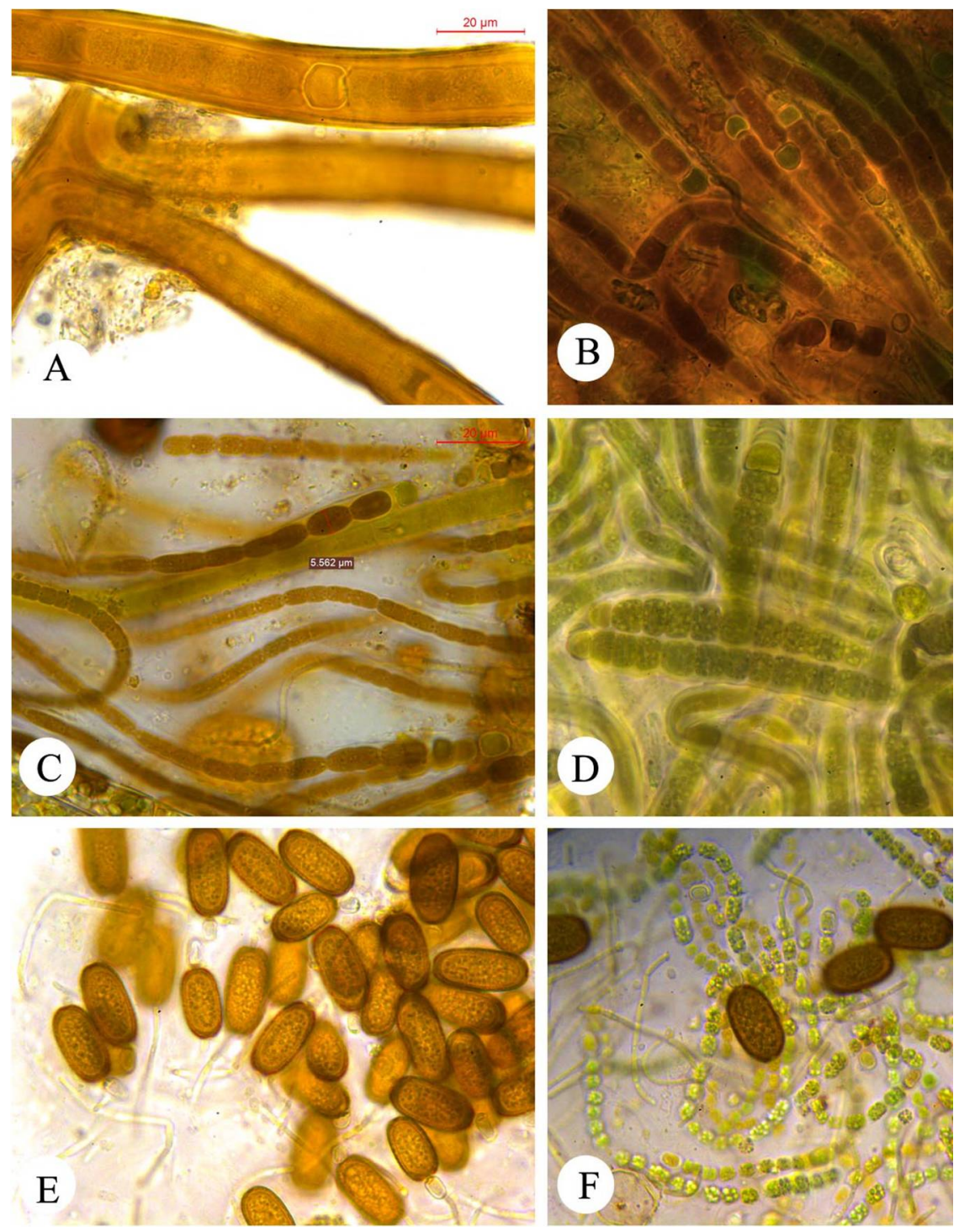

Fig. 2. A. Scytonema pascheri, B. Microchaete investiens, C. Microchaete species 1, D. Microchaete species 2, E,F. Cylindrospermum muscicola.

11. Microchaete species 1

(Fig. 2C)

Filaments long, $9.8 \mu \mathrm{m}$ broad; trichome olive green, barrel shape, $3.5-7 \mu \mathrm{m}$ long, $5.8-7.8$ $\mu \mathrm{m}$ broad, broader than long towards the base; heterocyst basal, $4.8-6.5 \mu \mathrm{m}$ long, $5.5-6.7 \mu \mathrm{m}$ broad; sheath colourless, $1.72 \mu \mathrm{m}$ thick, slightly lamellated; granules prominent.

Ecology: Attached scum on stone

Specimen examined: Kozhikode District, Chembanoda, Kerala, India: 11 Mar 2019, V. Geethu, Cyanobacterial Diversity Division, University of Calicut, CU No: 167121. 


\section{Microchaete species 2}

(Fig. 2D)

Filaments straight or coiled, brown coloured, tip of the filament is rounded; trichome 6.1-6.8 $\mu \mathrm{m}$ broad; cells elongated and cylindrical, 3.6-16 $\mu \mathrm{m}$ long, 4-3 $\mu \mathrm{m}$ broad; heterocysts spherical to hemispherical, basal or intercalary, sometimes 2-3 heterocysts comes together, 5.3-6.0 $\mu \mathrm{m}$ long, 6.0-7.2 $\mu \mathrm{m}$ broad; sheath firm, thin, hyaline, unlamellated, close to the trichome; granules present.

Ecology: Attached scum on stone

Specimen examined: Kozhikode District, Peruvannamuzhi forest, Kerala, India: 13 Sep 2018, V. Geethu, Cyanobacterial Diversity Division, University of Calicut, CU No: 158537.

Family: Nostocaceae; Genus: Cylindrospermum Kützing ex É.Bornet et C. Flahault

13. Cylindrospermum muscicola Kutzing ex Bornet et Flahault

(Fig. 2E,F) (Kutzing in Phyc. germ., 173, 1845, Tab. Phycologie, 1, 53, pl. 98, fig. 1, 1849; Bornet et Flahault in Revision des Nostocacees heterocystees, 254, 1888; Forti in De Toni, Sylloge Algarum, 5, 477, 1907)

Trichome green, cylindrical or quadrate, 3-5 $\mu \mathrm{m}$ long, $3 \mu \mathrm{m}$ broad; heterocyst terminal, yellow, 3.2-5 $\mu \mathrm{m}$ long, 4-4 $\mu \mathrm{m}$ broad; granules present; akinete dark brown, 15-21 $\mu \mathrm{m}$ long, 8 $10 \mu \mathrm{m}$ broad; sheath absent.

Ecology: Seen on soil

Specimen examined: Kozhikode District, Peruvannamuzhi forest, Kerala, India : 21 Jan 2019, V. Geethu, Cyanobacterial Diversity Division, University of Calicut, CU No: 167109.

Distribution: Kerala, Peruvannamuzhi forest, India; Britain, Czech Republic, Germany, Greece, Romania, Russia, Slovakia, Spain; North America, Arkansas,Cuba; South America: Argentina, Brazil,Sudan,Bangladesh, India, Iraq, Israel, Pakistan,China, Tajikistan, Japan, Russia, Pacific

14. Desmonostoc muscorum (Agardh ex Bornet et Flahault) Hrouzek et Ventura

(Fig. 3A) (Hrouzek et al. in Fottea, Olomouc, 13:(2), 211, 2013; Whitton et al. in A coded list of freshwater algae of the British Isles, 2nd edn, 2003)

Trichome irregularly flexous, green; cells barrel shaped, granulated, $4.7 \mu \mathrm{m}$ broad, $4.6-6.3$ $\mu \mathrm{m}$ long; heterocyst rounded or hemispherical, pale green, terminal or intercalary, $4.3-5 \mu \mathrm{m}$ long, $3.5-4.3 \mu \mathrm{m}$ broad; akinetes oval, $8-12 \mu \mathrm{m} \times 4-8 \mu \mathrm{m}$.

Ecology: Seen on soil

Specimen examined: Kozhikode District, Peruvannamuzhi forest, Kerala, India: 21 Nov 2018, V. Geethu, Cyanobacterial Diversity Division, University of Calicut CU No: 167103.

Distribution: Kerala, Peruvannamuzhi forest, India; Romania, Russia, Britain, Czech Republic, France, Ireland, Scandinavia, Slovakia, Spain,Israel, America, Argentina, Brazil, Bangladesh, India, Iraq, Pakistan,Japan, Nepal, Taiwan, Australia, The New Zealand, Ghana, Sudan.

15. Desmonostoc sp.

(Fig. 3B)

Trichome yellowish green, rounded, enclosed in a sheath, $5-8.3 \mu \mathrm{m}$ long, $5-7 \mu \mathrm{m}$ broad; heterocyst intercalary, elongated or oval, yellow, $7-11.5 \mu \mathrm{m}$ long, $6-7 \mu \mathrm{m}$ broad; sheath hyaline, colourless, $2-2.5 \mu \mathrm{m}$ thick.

Ecology: Attached to stone. 

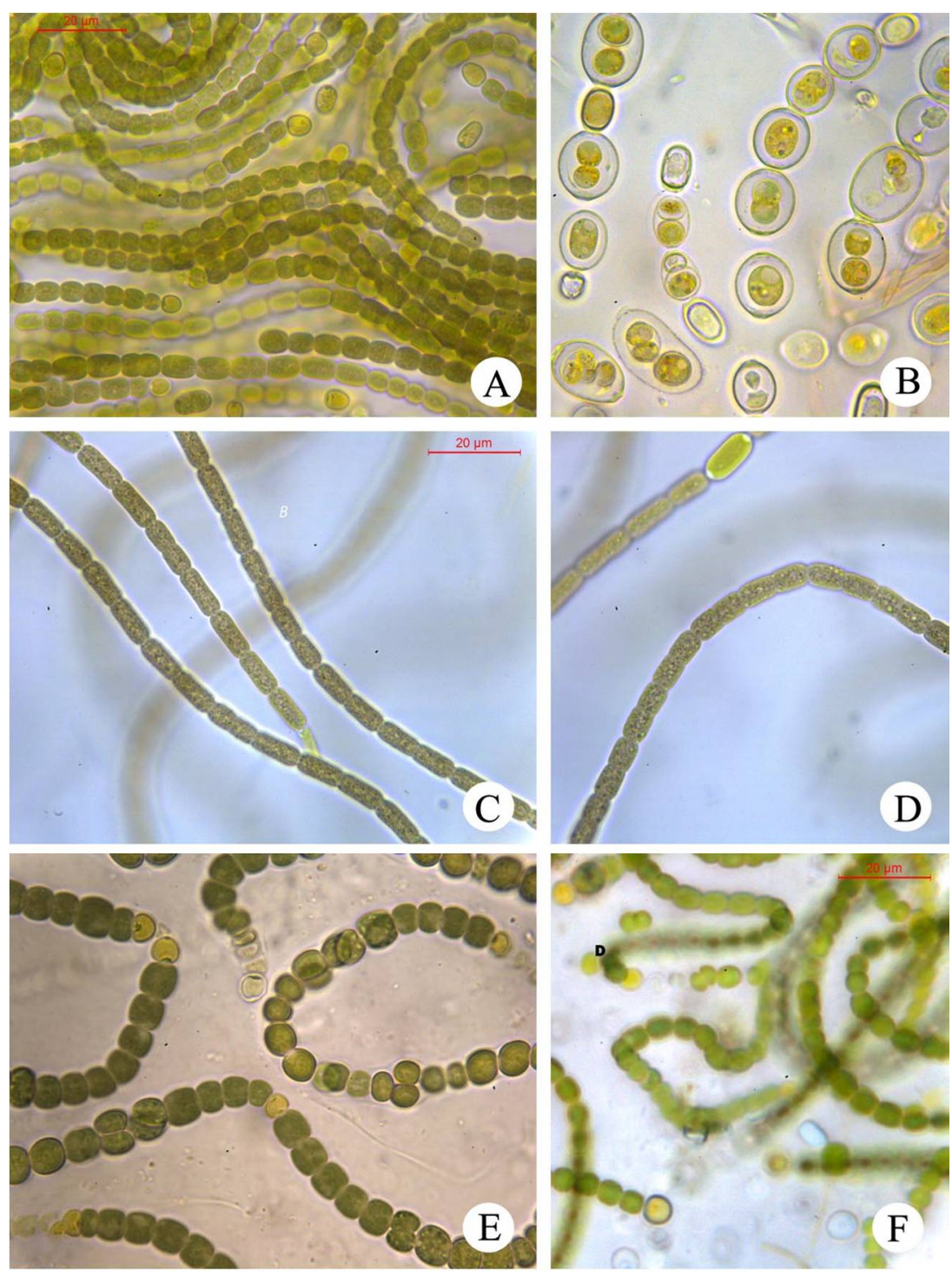

Fig. 3 A. Desmonostoc muscorum, B. Desmonostoc sp., C-D. Nostoc nylstromicum, E. N. commune, F. N. interbryum.

Specimen examined: Kozhikode District, Peruvannamuzhi forest, Kerala, india: 21 Nove 2018, V. Geethu, Cyanobacterial Diversity Division, University of Calicut, CU No: 167106.

16. Nostoc commune Vaucher ex Bornet et Flahault

(Fig . 3E)

Vaucher in Historie des Conferves d' eau douce, pl. 16, fig. 1, 222, 1803; Bornet et flahault in Revision des Nostocacees heterocystees, 203, 1888; Forti in De Toni, Sylloge Algarum, 5, 404, 1907 
Colonies macroscopic, gelatinous; trichome olive green; cells spherical or barrel shaped, 6.2 $7.8 \mu \mathrm{m}$ long, $6-8 \mu \mathrm{m}$ broad; heterocyst yellow, intercalary or terminal, intercalary ones spherical shaped, terminal one hemispherical, $5-7.2 \mu \mathrm{m}$ broad, $3.8-6.5 \mu \mathrm{m}$ long; granules absent.

Ecology: Attached to stone

Specimen examined: Kozhikode District, Peruvannamuzhi forest, Kerala, India: 13 Sep 2018, V. Geethu, Cyanobacterial Diversity Division, University of Calicut, CU No: 158539.

Distribution: Kerala, Peruvannamuzhi forest, India, Arctic, Ellesmere Island, Russia, Britain, Czech Republic, France, Georgia, Germany, Greece, Ireland, Italy, Lithuania, Romania,, Scandinavia, Slovakia, Spain, , Turkey, Alaska, Arkansas, Argentina, Ghana, Indian Ocean Islands, Rodrigues Island, Bangladesh, India, Iraq, Israel, Pakistan, Sri Lanka,Japan, Korea, Nepal, Russia Singapore, Vietnam,Australia and The New Zealand, Australia

\section{Nostoc nylstromicum Classenn}

(Fig. 3C,D)

(Claassen in Bothalia, 7: 3, pl. 2, fig. 2, 563, 1961; Komárek in Susswasserflora von Mitteleuropa, Vol. 19 pp. i-xviii, 1-1130, 2013)

Filaments yellowish brown; cells barrel or cylindrical, $2.3 \mu \mathrm{m}$ broad, $2.8-3.6 \mu \mathrm{m}$ long; heterocyst terminal, pale yellow in colour, $3.7 \mu \mathrm{m}$ long, $2.6 \mu \mathrm{m}$ broad.

Ecology: Attached to stone.

Specimen examined: Kozhikode District, Peruvannamuzhi forest, Kerala, India: 21 Nov 2018, V. Geethu, Cyanobacterial Diversity Division, University of Calicut, CU No : 167103.

Distribution: Kerala, Peruvannamuzhi forest, India.

Comment: The species has been reported as a first record in India.

18. Nostoc interbryum Sant'Anna et al.

(Fig. 3F)

(Anna et al. in Hoehnea, 34:1, fig. 1, 96, 2007; Werner in Catálogo de plantas e fungos do Brasil, 1, pp. 356-366, 2010; Komarek in Susswasserflora von Mitteleuropa. Cyanoprokaryota: 3rd part: heterocystous genera, 19, pp. i-xviii, 1-1130, 2013)

Filaments flexous, dark green; cells $4.6-5.2 \mu \mathrm{m}$ broad, $5 \mu \mathrm{m}$ long, rounded or spherical; heterocyst terminal, spherical, pale yellow, $5-5.5 \mu \mathrm{m}$ long , $4.5-5.6 \mu \mathrm{m}$ broad.

Ecology: Attached to soil.

Specimen examined: Kozhikode District, Peruvannamuzhi forest, Kerala, India: 21 Nov 2018, V. Geethu, Cyanobacterial Diversity Division, University of Calicut, CU No: 167103.

Distribution: Kerala, Peruvannamuzhi forest, India.

Comment: The species has been reported as a first record in India.

During the present study of filamentous cyanobacteria in Peruvannamuzhi forest and Janaki forest of Western Ghats, a total of 18 species belonging to 9 genera distributed in 4 families such as Oscillatoriaceae, Scytonemataceae, Microchaetaceae, Nostocaceae under 2 orders such as Oscillatoriales and Nostocales were identified. The diversity of filamentous cyanobacteria in this area is very high. Both heterocystous and non- heterocystous forms were equally dominating in this region were noticed in this study. About 10 heterocystous species under 5 genera were identified followed by 8 non-heterocystous species under 4 genera. They were found in aquatic, terrestrial and lithophytic habitats. But most of the sampling were done from rocky habitats. They are luxuriantly flourishing in these tropical forest because the litter as well as the alkaline $\mathrm{pH}$ of the soil greatly influences its abundance and diversity. In place like Kerala, the rainfall is always there around nine months in a year. This also greatly influence in the existence of these microorganisms under undisturbed evergreen forest of Western Ghats. Several studies were 
conducted about the tropical cyanobacteria and contributed much from Amazon forest of Brazil by Sant'Anna et al. (1991, 2007) and Branco et al. (2009)

Among heterocystous forms, the cyanobacterial genera Nostoc and Microchaete showed maximum abundance with 3 species each, followed by Desmonostoc with 2 species. The heterocystous genera, Cylindrospermum and Scytonema were represented by single species each and showed least abundance in the study area. Among non- heterocystous forms, the genus Oscillatoria showed maximum species richness with 4 species followed by Lyngbya with 2 species. The genera Planktothrix and Phormidium showed least abundance represented by single species each (Planktothrix planctonica and Phormidium retzi).

According to the present study diversity of filamentous forms were more in Peruvannamuzhi forest as compared to Janaki forest. About 15 taxa of cyanobacteria under 8 genera were identified from Peruvannamuzhi forest and 3 cyanobacterial taxa were identified from Janaki forest. Among 18 taxa 14 cyanobacterial taxa were identified up to species level the remaining 4 taxa were identified up to genus level. The species richness is very high in tropical forests such as Western Ghats. There are reports on cyanobacteria of Western Ghats of Maharashtra by Nikam et al. (2013), Many species found in this study were also distributed in other parts of the world. The cyanobacteria are highly adapted to these regions because they occur as thick leathery sheaths or mucilaginous masses which protects it from desiccation is also considered as an adaptation to this prokaryotic organisms.

\section{Conclusion}

The cyanobacterial taxonomy has been changing from time to time. Due to is microscopic and plasticity in its morphology it is very difficult in its identification, but owing to its varied application in many industries it is important to find out the undiscovered taxa especially from tropical forest because they are the biodiversity hotspots.In the present study we recorded eighteen cyanobacterial taxa out of which three taxa (Planktothrix planktonica, Oscillatoria euboeica and Nostoc interbryum are new to India). This study also helps to understand the geographical distribution as well as the type of habitat which will be beneficial for the further exploration of these organisms.

\section{Acknowledgement}

The authors thank the Head, Department of Botany, University of Calicut, Kerala, India for providing necessary facilities to carry out this research.

\section{Author contribution}

M. S. designed the research. G.V. contributed to data acquisition and collection. G.V. and M.S. analyzed and interpreted the results. G.V. and M.S. led the writing. Both authors revised and approved the manuscript.

\section{Additional information}

The authors declare no competing financial interests.

\section{References}

Anagnostidis, K and Komárek, J. 1988. Modern approach to the classification system of cyanophytes. 3. Oscillatoriales. Archiv für Hydrobiologie Supplement 80: 327-472.

Anagnostidis, K. 2001. Nomenclatural changes in cyanoprokaryotic order Oscillatoriales. Preslia Praha 73: 359-375. 
Anand, N. 1989. Hand book of blue green algae of rice field of South India. Bishen Singh Mahendra Pal Singh, pp. 20-45.

Ansari, Z., Tambe, S.S. and Nandan, S.N. 2012. Biodiversity studies of Cyanophyceae in Mausam River of Malegaon city, district: Nasik, state: Maharashtra. International Journal f Science \& Pharma Educational Research 2: 69-72.

Bhosale, L.J. and Dhumal, S.N. 2012. Fresh water phytoplankton from Gadhinglaj Tahsil of Kolhapur distrist, Maharashtra, India. Indian Hydrobiology 15(2): 153-165.

Branco, L.H.Z., Hoffmann, L., Teixeira, J.P., Ferreira, V. and Morais Filho, J.C. 2009. Aerophytic cyanoprokaryotes from Atlantic rainforest region of São Paulo State, Brazil: Chroococcales and Oscillatoriales. Cryptogamie, Algologie 30:135-152.

Chakraborty, T., Mukhopadhyay, A. and Pal, R. 2010. Micro algal diversity of Kolkata, West Bengal, India. Indian Hydrobiology 12(2): 204-224.

Chellappa, S., Chellappa, N. and Marinho, I.R. 2004. Freshwater phytoplankton assemblages and bloom of toxic cyanophyceae of Campo Grande reservoir of Rio Grande do Norte of Brazil. Indian hydrobiology 7: $151-171$.

Claassen, M. I. 1961. A contribution to our knowledge of the freshwater algae of the Transvaal Province. Bothalia 7: 559-666.

Datta, S. and Keshri, J.P. 2014. Soil and sub aerial blue green algae (Cyanoprokaryotes) of Burdwan, West Bengal. Vegetos 27(2): 112-126.

Desikachary, T.V. 1959. Cyanophyta. Indian Council of Agric. Research, New Delhi. 686 pp.

Dey, N. 2012. The different members of Chroococcales, The Blue-green algae from Darjeeling Himalayas, India. J. Econ. Taxon. Bot. 36(1): 216-222.

Dhingra, R. and Ahluwalia, A.S. 2007a. Cyanostylon Geitler (Cyanophyta) from Punjab, India. J. Ind. Bot. Soc. 86(3\&4): 22-24.

Dhingra, R. and Ahluwalia, A.S. 2007b. Genus Phormidium Kutzing Ex. Gomont Cyanoprokaryote) from diverse habitats of Punjab. J. Ind. Bot. Soc. 86(3\&4): 86-94.

Gomont, M. 1892. Monographie des Oscillariées (Nostocacées Homocystées). Deuxième partie. Lyngbyées. Annales des Sciences Naturelles, Botanique, Série 7(16): 91-264

Gupta, P. 2012. Algae of India. A Checklist of Cyanoprokaryota (Cyanophyceae). Salt Lake, Kolkata: Botanical Survey of India.

Gupta, R.K., Kumar, M. and Paliwal, G.S. 2006. Glimpses of Cyanobacteria. India: New Delhi, Daya Publishing House.

Halder, N. and Sinha, S.N. 2013. Diversity of the genera Gloeotrichia Agardh and Rivularia (Roth) Agardh from Hooghly district of West Bengal, India. I. J. Fund. Appl. Life Sci 3(3): 29-35.

Halder, N. 2016. Chemical composition and antibacterial activity of Lyngbya major Menegh. ex Gomont. Appl. Sci. Rep. 13: 25-28.

Halder, N. 2015. Recollections and taxo-ecological studies of Coleochaete scutata Bréb., Coleochaete pseudosoluta Gauthier-Lièvre and Coleochaete conchata Möb., West Bengal, India. Nepalese J. Biosci. 5: 21-25.

Hoek, V.D.C., Mann, D.G. and Johns, H.M. 1993. Algae: An Introduction to Phycology. 2nd ed. Cambridge University Press, Cambridge.

Hrouzek, P., Lukešová, A., Mareš, J. and Ventura,S. 2013. Description of the cyanobacterial genus Desmonostoc gen. nov. including D. muscorum comb. nov. as a distinct, phylogenetically coherent taxon related to the genus Nostoc. Fottea, Olomouc 13(2): 201-213.

John, J. and Francis, M.S. 2013. An Illustrated Algal Flora of Kerala. Vol-1, Idukki Distrtict. Pranatha Books, Cochin, pp. 245-281.

Keshri, J.P. and Chatterjee, S. 2010. First record of two cyanoprokaryotas,Oscillatoria (Oscillatoriales) and Nostoc (Nostocales), endophytic within the angiosperm Alternanthera sessilis (Amaranthaceae) from India. Alogological Studies 135: 83-88. 
Komárek, J. and Anagnostidis. 2005. Süsswasserflora von Mitteleuropa. Cyanoprokaryota: 2. Teil/2nd Part: Oscillatoriales. München: Elsevier Spektrum Akademischer Verlag 19: 1-759.

Komarek, J., Kastovsky, J., Mares, J. and Johansen, J. 2014. Taxonomic classification of cyanoprokaryotes (cyanobacterial genera) 2014, using a polyphasic approach. Preslia 86: 295-335.

Mishra, U. and Pubbi. 2004. Cyanobacteria. A Potential Biofertilizer for Rice, Resonance, Genral Article, pp. $6-10$.

Naskar, N., Naskar, K.R. and Sen, C.R. 2008. Brakish water Oscillatoriaceae North 24-Parganas, West Bengal, India. Bangladesh J. Plant Taxon. 15(1): 31-38.

Nikam, T.D., Nehul, J.N., Gahile, Y.R., Auti, B.K., Ahire, M.L., Nitnaware, K.M., Joshi, B.N. and Jawali, N. 2013. Cyanobacterial diversity in Western Ghats region of Maharashtra, India. Bioremediation, Biodiversity and bioavailability, pp. 71-80.

Pandey, V.D., Pandey, A. and Sharma, V. 2013. Biotechnological application of Cyanobacterial Pycobiliproteins. Int. J. Curr Micobiol. App. Sci. 2(9): 89-97.

Rippka, R., Deruelles, J., Waterburg, J.B., Heedman, M. and Stainer, R.Y. 1979. Genetic assignments, strain histories and properties of pure culture of cyanobacteria. Journal of General Microbiology 111(15): $1-61$.

Roy, S., Debnath, M. and Ray, S. 2014a. Cyanobacterial flora of the geothermal spring at Panifala, West Bengal, India. Phykos 44(1): 1-8.

Roy, S., Bhattacharya, S., Debnath, M. and Ray, S. 2014b. Diversity of cyanobacterial flora of Bakreswar geothermal spring, West Bengal, India-II. Algological Studies 147: 29-44.

Sant'Anna, C.L., Azeved, O.M.T.P., Branco, L.H.Z. and Komárek, J. 2007. New aerophytic morphospecies of Nostoc (Cyanobacteria) from São Paulo State, Brazil. Hoehnea 34: 95-101.

Sant'Anna, C.L., Silva, S.M.F. and Branco, L.H.Z. 1991. Cyanophyceae da Gruta-que-Chora, município de Ubatuba, Estado de São Paulo, Brasil. Hoehnea 18: 75-97.

Thajuddin, N., Nagasathya, A., Chelladevi, R. and Saravanan, P. 2002. Biodiversity of cyanobacteria in different salt pans of Pudukkottai District, Thamilnadu. Seaweed Research and Utilization 24: 1-11.

Tiwari, A. and Chauhan, S.V.S. 2006. Seasonal variation in some Oscillatoria species from polluted ponds of Agra. J. Ind. Bot. Soc. 85: 110-117.

Tiwari, A. and Chauhan, S.V.S. 2008. Growth and periodicity of cyanobacterial bloom in a polluted pond of Agra city. J. Environ. Biol. 29(6): 859-862. 